\title{
Chicken Swarm Optimization for PTS based PAPR Reduction in OFDM Systems
}

\author{
Saruti Gupta, and Ashish Goel
}

\begin{abstract}
Partial transmit sequence (PTS) is a well-known PAPR reduction scheme for the OFDM system. One of the major challenge of this scheme is to find an optimal phase vector using exhaustive search over all the allowed phase factor combinations. This leads to increased search complexity which grows exponentially as the number of sub-blocks is increased. In this paper, chicken swarm optimization (CSO) based PTS system is designed that aims to find an optimal solution in less number of average iterations and therefore results in reduced computational complexity of the system. We have proposed two categories of the algorithm: (i) CSO-PTS system without threshold limit on PAPR (ii) CSO-PTS system with threshold limit on PAPR. Both the schemes offer effective trade-offs between the computational complexity and the PAPR reduction capability of the system. Simulation results confirm that our proposed schemes perform well in terms of low computational complexity, lesser number of average iterations and improved PAPR reduction capability of the OFDM signal without any loss in BER performance of the system.
\end{abstract}

Index terms - orthogonal frequency division multiplexing (OFDM), peak-to-average power ratio (PAPR), partial transmit sequence (PTS), chicken swarm optimization (CSO).

\section{INTRODUCTION}

Orthogonal frequency division multiplexing (OFDM) is a multicarrier modulation technique that is widely adopted in high speed wireless applications such as European standard for audio and video broadcasting like digital video broadcasting (DVB), wireless network standard like IEEE 802.11a and IEEE 802.16d based WiMax broadband access. The main advantage of this multicarrier technique is the high data rate and immunity against multipath propagation effects. But one of the major drawback of the OFDM system is the high peak-to-average power ratio (PAPR) of the OFDM signal that affects the system performance and leads to in-band distortions and out-of-band radiations [1]. Therefore, it is necessary to limit the PAPR of the OFDM signal so that the system performance will not degrade, when amplified by nonlinear HPA. Various signal distortion and signal

Manuscript received January 17, 2020; revised May 26, 2020. Date of publication July 15, 2020. Date of current version July 15, 2020. The associate editor prof. Gordan Šišul has been coordinating the revew of this manuscript and approved it for publication.

Authors are with the Electronics \& Comm. Engineering Department, JIIT, Noida (e-mails: sarutigupta11@gmail.com,ashish.goel@jiit.ac.in).

Digital Object Identifier (DOI): 10.24138/jcomss.v16i3.999 scrambling schemes have been proposed in the literature for PAPR reduction of the OFDM signal. Signal distortion schemes consist of clipping [2], clipping and filtering [3] and companding [4]. Signal scrambling schemes includes coding [5], selective mapping (SLM) [6], partial transmit [7], active constellation extension (ACE) [8], tone Reservation TR [9] and tone injection TI [10]. PTS is a well-known distortionless PAPR reduction technique, where frequency domain OFDM is divided into signal sub-blocks and these sub-blocks are optimally combined using phase factors to obtain OFDM signal with minimum PAPR. These sub-blocks after taking IFFT are rotated by the phase factors resulting in different partial sub sequences and further they are merged to obtain a time domain OFDM signal. Among them, the one with lowest PAPR value that corresponds to an optimal phase is selected for transmission. The major concern in this existing scheme is to find an optimal phase factor by performing exhaustive search on all the possible phase combinations which grow exponentially as the number of sub-blocks increases [11]. This leads to high computational complexity of the system and more processing time for evaluations. In the PTS scheme, optimization of phase factor plays a vital role for obtaining better PAPR values of the OFDM signal and low computational complexity of the system.In the literature various meta-heuristic algorithms have been implemented for PTS-OFDM system to achieve the optimization of the phase factors. Some of these optimization algorithms namely Differential Evolution (DE) [12], Genetic Algorithm (GA) [13], Artificial Bee Colony (ABC) algorithm [14], shuffled frog leaping algorithm [15], and hybrid PS-GW [16] have been proposed for PTS-OFDM scheme to find optimal phase factors. These offer low computational complexity with improved PAPR reduction of the OFDM signals. But their low convergence performance in terms of speed and precision are the limiting factor for finding the optimal solution. Few nature-inspired optimization algorithms such as particle swarm optimization (PSO) [17], firefly algorithm [18] and Galactic Swarm Optimization (GSO) [19] are also used with the PTS-OFDM system. But they do not exhibit effective trade-offs between the computational complexity and PAPR reduction capability of the OFDM signals. A suitable optimization algorithm for the PTS technique should have the capability to achieve an optimal solution with low complexity and better convergence performance.

In recent years chicken swarm optimization (CSO) algorithm suggested in [20] has drawn the attention of many 
researchers for finding the optimal solutions in various engineering applications. It can be studied from the literature that CSO outperforms to solve optimization problems with better convergence performance in terms of speed and accuracy. In [21], an improved CSO algorithm is proposed for analysing optimization capabilities on various benchmark functions in high dimensions. As investigated in [22] the designed CSO algorithm is capable of solving constrained based optimization problems with fast convergence rate and high precision in both low and high dimensions. In [23] CSO algorithm is used with CO-OFDM system to achieve an optimal phase solution for the PAPR reduction of OFDM signals. Implementation of CSO with PTS-OFDM system is proposed in [24] that reduces PAPR of OFDM signals for different number of sub-carriers, different sub-blocks and empirical parameters of the algorithm. Scope of [24] is limited to the evaluation of PAPR of the OFDM signals without any analysis of the computational complexity and study of the receiver side performance.

In this paper, CSO based PTS-OFDM system is proposed to improve the PAPR reduction capabilities of the OFDM signal with low computational complexity to find optimal phase factors. Here, we have proposed two schemes using CSO based PTS system (i) CSO-PTS system without threshold limit on PAPR (ii) CSO-PTS system with threshold limit on PAPR. The designed schemes offer effective tradeoffs between the computational complexity and PAPR reduction of the OFDM signal. Proposed schemes find an optimal solution with less number of average iterations in comparison to conventional PTS-OFDM system. The significant novelty of our designed scheme is the implementation of the threshold based criteria that results in low computational complexity, lesser number of average iterations per OFDM frame and improved PAPR reduction of the OFDM signals without any BER degradations over AWGN and fading channels.

This paper is organized as follows: In section II the conventional PTS-OFDM system is discussed. The proposed chicken swarm optimization (CSO) based PTS-OFDM system is investigated in Section III. Simulation results in terms of PAPR reduction, average number of iterations, CCRR value and BER performance are presented in Section IV. Conclusions are drawn in Section V.

\section{CONVENTIONAL PTS-OFDM SYSTEM}

Consider an OFDM data block $X=\left[X_{0}, X_{1}, \ldots \ldots . X_{N-1}\right]$ that consists of $N$ complex input data symbols. During their parallel transmission over the $N$ subcarriers, each of these subcarriers is modulated using using $\mathrm{M}$-ary phase-shift keying (M-PSK) or M-ary quadrature amplitude modulation (MQAM).

The time domain OFDM signal sequence after applying the inverse fast Fourier transform (IFFT) to modulated block is given as:

$$
x(n)=\frac{1}{\sqrt{N}} \sum_{k=0}^{N-1} X_{k} \exp \left(\frac{j 2 \pi n k}{N}\right), \quad 0 \leq n \leq N-1
$$

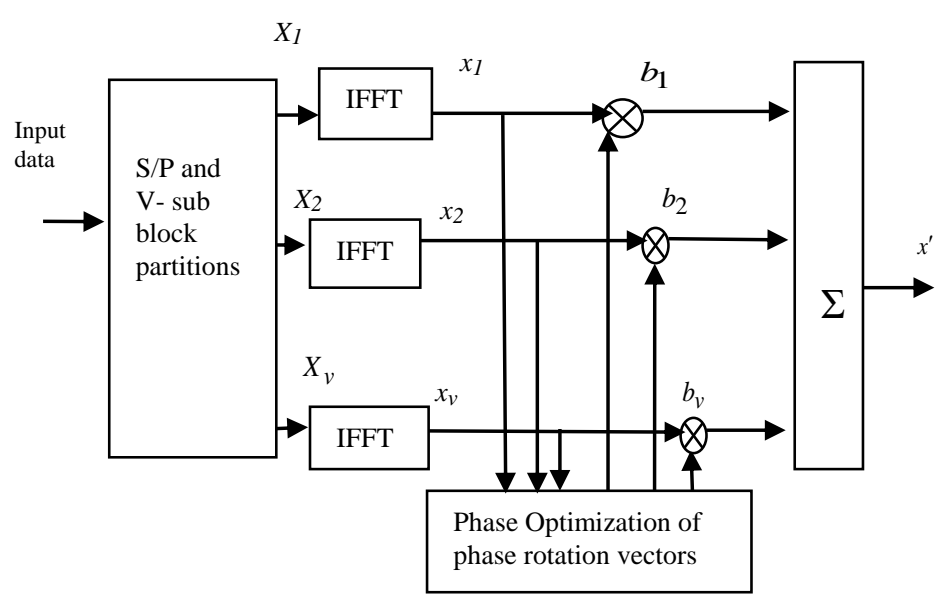

Fig. 1. Block diagram of conventional PTS-OFDM system.

The PAPR of the OFDM signal $x(n)$ is defined as the ratio of the peak power and the mean power which can be expressed as

$$
P A P R=\frac{\max _{n \in[0, N-1]}\left\{\left|x_{n}\right|^{2}\right\}}{E\left\{\left|x_{n}\right|^{2}\right\}}
$$

where $E\{$.$\} is the expectation operator.$

To measure the PAPR reduction capability, complementary cumulative distribution function (CCDF) is used as an efficient parameter. It is defined as the probability of an OFDM signal with PAPR value that exceeds a specific threshold value of $\mathrm{PAPR}$ and is given as:

$$
C C D F\left(\gamma_{0}\right)=\operatorname{Pr} o b\left\{P A P R>\gamma_{0}\right\}
$$

In the conventional PTS-OFDM system the input block $X$ is split as $X_{v}=\left[X_{v, 0}, X_{v, 1}, \ldots \ldots . X_{v, N-1}\right]$ such that $X=\sum_{v=0}^{V-1} X_{v}$ where $V$ is the number of disjoint sub-blocks. After applying the IFFT on each of these sub-blocks, the time domain partial sequences are rotated by the phase weighting factors $b_{v}, v=0$, $1, \ldots . . V-1$ having unit magnitude that is restricted to $\{ \pm 1\}$ or $\{ \pm 1, \pm j\}$ for $W=2$ and $W=4$ respectively. On combining these partial sequences, we obtain the signal sub-sequence $x$ ' as follows:

$$
x^{\prime}=\sum_{v=0}^{V-1} b_{v} x_{v}
$$

where $\quad b_{v}=e^{j \phi_{v}}, \quad 0 \leq \phi_{v} \leq 2 \pi \quad$ for $\quad v=0,1, \ldots V-1 \quad$ and $x^{\prime}=\left[\mathrm{x}_{0}^{\prime}, \mathrm{x}_{1}^{\prime}, \ldots \ldots . \mathrm{x}_{N-1}^{\prime}\right]$ 
The principle of this technique is to find an optimal phase vector out of all $2^{V-1}$ available phase factor combinations, which achieves a minimum PAPR value among the possible signal sub-sequences. The objective function of the optimal phase vector corresponding to the minimum PAPR value can be expressed as:

Minima:

$$
f(b)=\frac{\max \left\{\left|x^{\prime}(\mathrm{b})\right|^{2}\right\}}{E\left\{\left|x^{\prime}(\mathrm{b})\right|^{2}\right\}}
$$

In the conventional PTS technique $2^{V-1}$ phase combinations are searched to find the minimum PAPR value of the OFDM signal. But the major concern is the number of searches required to select an optimal phase vector that grows as the number of sub blocks is increased for better PAPR performance [24]. This leads to more number of exhaustive searches and increased computational complexity. To achieve PAPR reduction of the OFDM signal with low computational complexity, we have proposed CSO based PTS schemes that overcome the discussed shortcomings of the conventional PTS-OFDM system.

\section{PROPOSED SCHEMES BASED ON CSO}

\section{A. Chicken Swarm Optimization Algorithm}

Chicken swarm optimization (CSO) is a bio-inspired algorithm that imitates the behavior of chicken swarm hierarchically to find an optimal solution [20]. The optimization of the objective function is based on the cooperative nature of the swarm individuals namely head rooster, hens and chicks. Following steps are involved for the optimization process:

- Swarm population consists of total $p$ chickens that are divided into different groups. Each group consists of a head rooster, some hens and few chicks which search for food in a hierarchical order. Let's consider number of rooster, hens and chicks as $R N, H N$ and $C N$.

- The roles of the swarm in each group are decided on the basis of designed fitness function. Those with the best and worst fitness values act as rooster and hens respectively while rest of the swarm is referred as chicks. Random mother-child relationship is established between the mother hens $M N$ and the chicks $C N$.

- In a given time frame, each relation remains constant for every status update $\left(G_{s}\right)$ in a hierarchical order.

- In a competition to search for food each head rooster in a group is assisted by the dominant hens while the chicks would depend on their mother hens for food. Also the dominant chicken moves along with the head rooster and suppress the submissive ones in search of food.

Then, solution is updated for each individual in a swarm while they search for the food. If the new solution is better than the previous ones, then it is replaced with the new solution until a termination condition is met.

\section{B. CSO-PTS System}

Considering the CSO-PTS system, we initialize the parameters namely maximum generations $G$, population size $p$, number of roosters $R N$, number of hens $H N$, number of chicks $C N$, number of mother hens $M N$, and number of updates $G_{s}$. In a D-dimensional search space the food source position for the rooster corresponding to the phase vector $b_{i}=\left[b_{1}, b_{2}, \ldots . . b_{K}\right]$ is given as

$$
b_{i, K}^{t+1}=b_{i, \mathrm{~K}}^{t}\left(1+\operatorname{Randn}\left(0, \sigma^{2}\right)\right), \quad 1 \leq K \leq D
$$

$$
\sigma^{2}=\left\{\begin{array}{ll}
1, \quad \text { if } f_{i} \leq f_{k} \\
\exp \frac{f_{k}-f_{i}}{|f|+\varepsilon}, & \text { otherwise }
\end{array}\right\}, \quad k \in\{1, p\}, 1 \leq i \leq p
$$

where $\operatorname{Randn}\left(0, \sigma^{2}\right)$ is a Gaussian distribution function, $\varepsilon$ is a small constant and $k$ is a rooster index which is randomly chosen.

The source position of food for the hen following the head rooster in terms of phase vector is presented as:

$$
b_{i, K}^{t+1}=b_{i, \mathrm{~K}}^{t}+S_{1} * \operatorname{Rand}^{*}\left(b_{\mathrm{r} 1, K}^{t}-b_{i, \mathrm{~K}}^{t}\right)+\mathrm{S}_{2} * \operatorname{Rand}^{*}\left(\mathrm{~b}_{\mathrm{r} 2, K}^{t}-b_{i, K}^{t}\right)
$$

$$
\begin{gathered}
\mathrm{S}_{1}=\exp \frac{f_{i}-f_{r 1}}{a b s\left(f_{i}\right)+\varepsilon} \\
\mathrm{S}_{2}=\exp \left(\left(f_{r 2}-f_{i}\right)\right)
\end{gathered}
$$

where $r_{l}$ is the rooster index corresponding to $i^{\text {th }}$ hen's groupmate and $r_{2}$ is an index of chicken (rooster or hen).

The chick's food source position looking for their mother hen can be written as:

$$
b_{i, K}^{t+1}=b_{i, \mathrm{~K}}^{t}+F L^{*} \operatorname{Rand}^{*}\left(b_{\mathrm{mth}, K}^{t}-b_{i, \mathrm{~K}}^{t}\right)
$$

where $b_{\text {mth, } K}^{t}$ chick's mother position and $F L$ is a parameter that is used to denote that the chicks would accompany their mother for food.

The food source position vector is defined as:

$$
b_{i, K}^{t+1}= \begin{cases}1 \quad \text { if } \quad 0 \leq b_{i, K}^{t+1} \leq \pi / 4 \\ -1 \quad \text { else }\end{cases}
$$

The fitness value function in the given swarm population can be computed as:

$$
F\left(b_{i, K}^{t+1}\right)=\frac{1}{1+f\left(b_{i, K}^{t+1}\right)}
$$


This function corresponds to the ones with the minimal values of the objective function $f(b)$ and hence represents the optimized search solution. In the entire swarm population, the comparisons with either local minima or with specific threshold of the fitness function are done and solutions in terms of optimal phase vector are updated accordingly.

In order to achieve an optimal value of the solution, some considerations based on the experimental trials conducted on the various benchmark functions in [21] are used for initialization of the parameters as follows: (i) Number of hens should be greater than number of roosters and number of chicks in each group; (ii) The mother hens are chosen randomly from the given number of hens; (iii) values of parameter FL lies in [0.4,1] and status update $G_{s}$ are chosen using $G_{s} \in[2,20]$.

The two proposed CSO-PTS Algorithms are designed below:

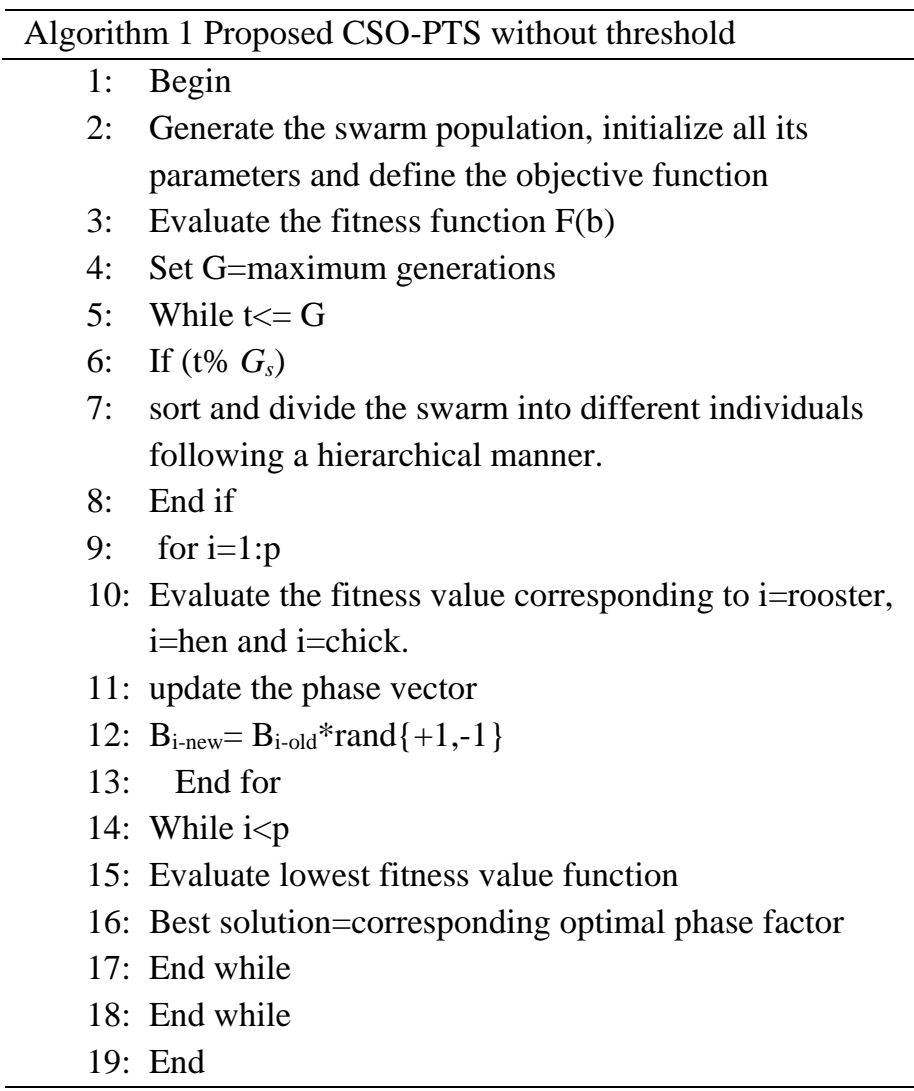
8: End if
9: for $\mathrm{i}=1: \mathrm{p}$
10: Evaluate the fitness value corresponding to $i=$ rooster, $\mathrm{i}=$ hen and $\mathrm{i}=$ chick.
11: update the phase vector
12: $\mathrm{B}_{\text {i-new }}=\mathrm{B}_{\mathrm{i} \text {-old }} *$ rand $\{+1,-1\}$
13: $\mathrm{PAPR}=$ fitness value
14: End for
15: While $\mathrm{i}<\mathrm{p}$ or PAPR $>\mathrm{T}$
16: Evaluate lowest fitness value function
17: Best solution=corresponding optimal phase factor
18: End while
19: End while
20: End

In CSO algorithm the randomly generated phase factor confined to size $p$, computes the optimal PAPR corresponding to the minimal fitness value function. In proposed Algorithm 1 , local minimum is updated for each iteration count and to evaluate the optimal value of PAPR all the searches are done till the maximum number of generation $G$. Whereas in Algorithm 2, we apply a threshold limit to the local minima. Here, rather than iterating up to the maximum generation, as the fitness value falls below a specific threshold the search is terminated. If the threshold value is small, the Algorithm 2 searches more combinations while for high threshold value, less number of search combinations are needed comparatively. Thus, there occurs a trade-off between the minimal PAPR solution and number of iterations required to search. Thus, it is observed that in Algorithm 1 more number of iterations is required as compared to Algorithm 2.

\section{Computational Complexity Analysis}

The computational complexity of our proposed scheme is evaluated in terms of real additions and real multiplications that are required for $N$-point IFFT computations and PAPR evaluations. For $V$ sub-blocks, we need $V N \log _{2} N$ complex additions and $\frac{V N}{2} \log _{2} N$ complex multiplications.

The computational complexity of a complex multiplication can be presented as four real multiplications and two real additions whereas a complex addition corresponds to two real additions. Hence $3 V N \log _{2} N$ real additions and $2 \mathrm{VN} \log _{2} N$ real multiplications are needed. Further $N(V-1)$ complex additions are required for each phase sample and for the PAPR computations $N$ real additions and $2 N$ multiplication are needed [25].

In the conventional PTS system, the numbers of samples searched are $2^{V-1}$. Thus, the complexity in terms of real additions $Z_{a d d}$ and real multiplications $Z_{m u l}$ can be represented as:

$$
\begin{aligned}
& Z_{\text {add }}=3 V N \log _{2} N+2\left(2^{V-1}\right)(V-1) N+2^{V-1} \\
& Z_{\text {mul }}=2 V N \log _{2} N+2^{V-1}(2 N)
\end{aligned}
$$


The computational complexity of our proposed scheme in terms of real additions $Z_{\text {add }}^{\prime}$ and real multiplications $Z_{m u l}^{\prime}$ can be represented as

$$
\begin{aligned}
& Z_{\text {add }}^{\prime}=3 V N \log _{2} N+2 A I(V-1) N+(A I) N \\
& Z_{\text {mul }}^{\prime}=2 V N \log _{2} N+A I(2 N)
\end{aligned}
$$

where $A I$ are the average iterations needed for generated samples.

Computational complexity reduction ratio (CCRR) is an effective parameter to compute the reduction in complexity of our proposed scheme with the conventional PTS scheme.

It can be represented as

$$
C C R R=\left(1-\frac{\text { complexityof the proposed scheme }}{\text { complexityof theconventional scheme }}\right) \times 100 \%
$$

High value of $C C R R$ results in lower complexity of the designed system.

\section{SIMULATION RESULTS}

Using various computer simulations, we evaluate the performance of our proposed system in terms of number of computations required for optimal search, PAPR improvements and BER performance over AWGN and Multipath Rayleigh fading channels. Simulations are done for 5000 OFDM symbols using QPSK modulation with $N=256$ subcarriers. Adjacent partitioning scheme for $V=8$ sub-blocks and weighting factor $W=2$ is used. Results are evaluated on the basis of different values of maximum generations $G$, population size $p$ and threshold limits to study the system performance. In our simulation we have set $R N=0.15^{*} p$, $H N=0.7^{*} p, M N=0.2^{*} p$, and $G_{s}=12$ to obtain the optimum value of our objective function in the proposed scheme. The complete specification of the simulation parameters is summarized in Table I.

TABLE I

SPECIFICATION OF SIMULATION PARAMETERS

\begin{tabular}{|l|l|}
\hline Parameters & Value \\
\hline OFDM symbols & 5000 \\
\hline Subcarriers & 256 \\
\hline Sub-blocks $(M)$ & 8 \\
\hline Modulation Used & QPSK \\
\hline Status Update $\left(G_{s}\right)$ & 12 \\
\hline population size $(p)$ & 10,15 and 20 \\
\hline Number of roosters $(R N)$ & $0.15^{*} p$ \\
\hline Number of hens $(H N)$ & $0.7^{*} p$ \\
\hline Number of mother hens $(M N)$ & $0.2^{*} p$ \\
\hline Channel Model & $\begin{array}{l}\text { AWGN, Multipath Rayleigh } \\
\text { fading (3-tap,SUI-5) }\end{array}$ \\
\hline
\end{tabular}

In Fig. 2, we have evaluated the CCDF of PAPR to study effect of maximum number of generations. Here, we have shown its effect by varying the maximum generations for
$G=5,10$ and 15 in the proposed algorithm. As noted from the curves on increasing the value of $G$, the PAPR performance is improved but at the cost of increased number of iterations to find the optimal phase factors. Hence, there occurs a trade-off between the PAPR reduction capabilities and computational complexity. We examine that for $G=15$ and at $\mathrm{CCDF}=10^{-3}$, proposed approach achieves about $3.1 \mathrm{~dB}$ PAPR reduction as compared to the original OFDM signal. Also, there is slight gap of $0.1 \mathrm{~dB}$ between the PAPR performance of the conventional PTS and proposed CSO-PTS system at $G=15$. But it requires less number of average iterations for optimal search in contrast to the conventional PTS systems.

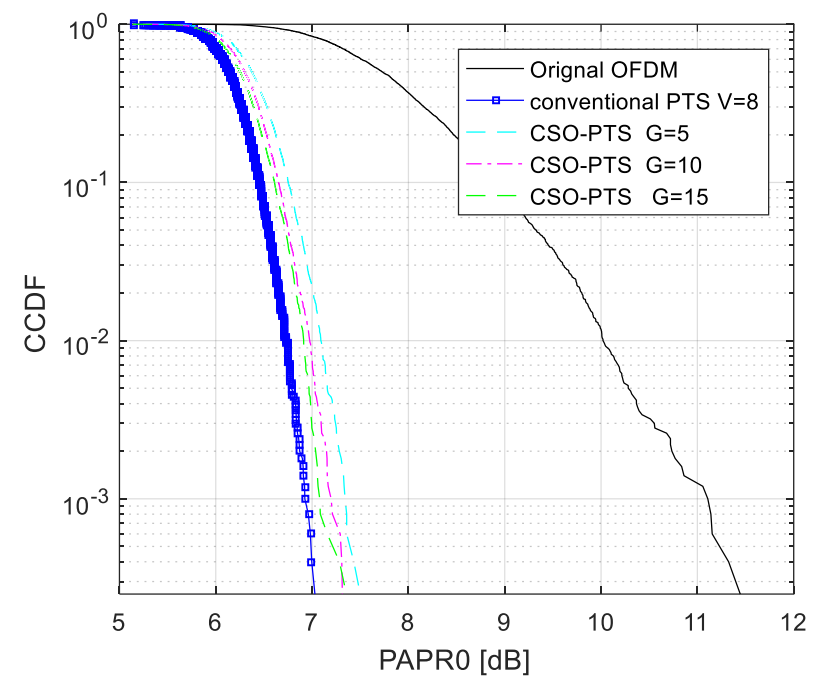

Fig. 2. PAPR performance of proposed scheme for different values of maximum generations.

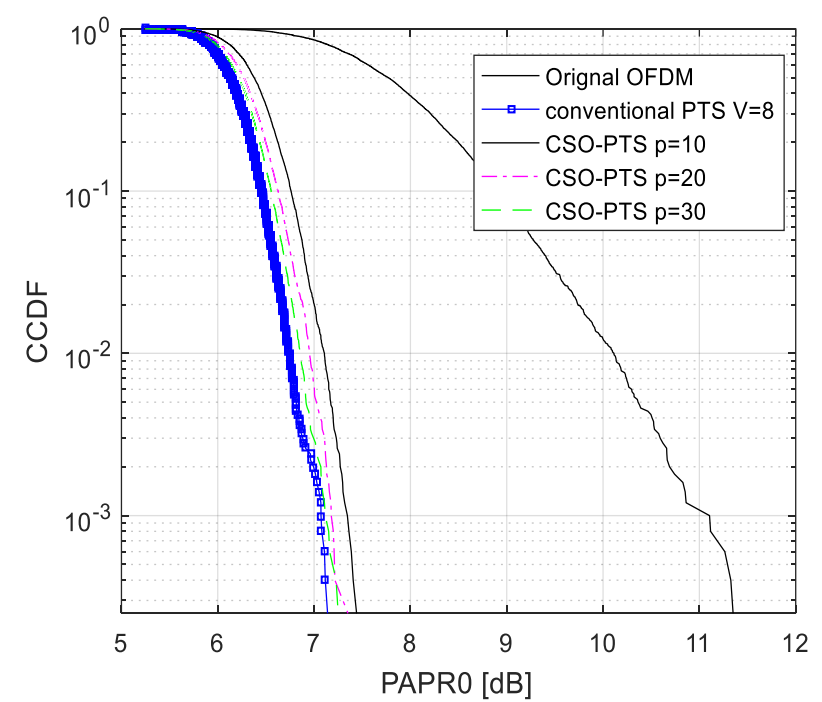

Fig. 3. PAPR performance of proposed scheme for different values of population sizes.

Fig. 3 shows the PAPR performance of proposed scheme for different values of population size $p=10,15$ and 20. It can be observed from the graph that the PAPR value of the OFDM signal is improved as we increase the size of swarm population. It is also noted that the average number of iterations required to search optimal phase factor combination 
increases for higher values of $p$. At a given $\mathrm{CCDF}=10^{-3}$ the proposed scheme achieves about 3.2dB PAPR reduction capability in comparison to original OFDM signal. Slight degradation in PAPR performance of $0.97 \mathrm{~dB}$ is observed, when we compare the proposed scheme with the conventional PTS scheme. But at the same time the proposed scheme requires lesser number of average iterations that leads to low computational complexity.

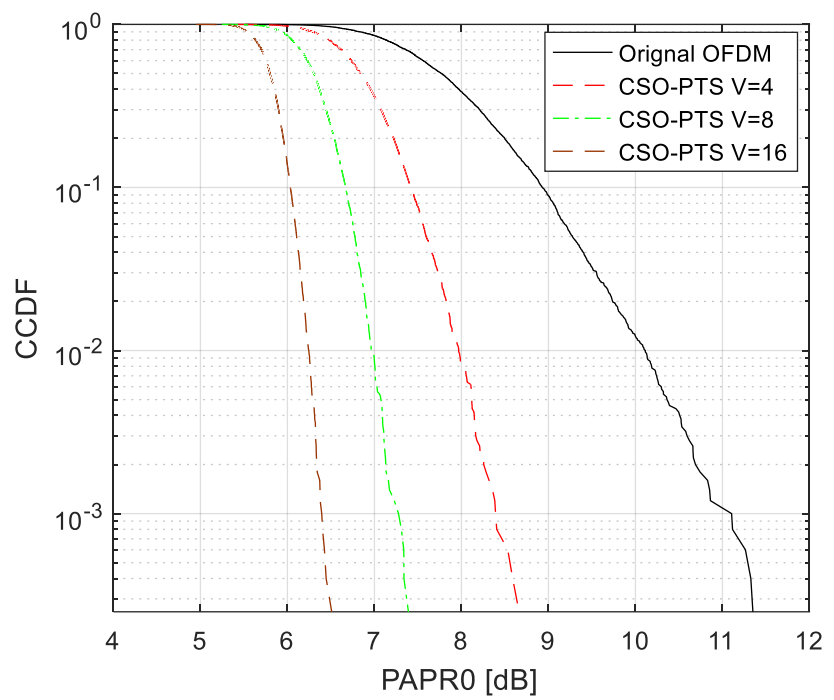

Fig. 4. PAPR performance of proposed scheme for different values of subblocks $\mathrm{V}=4,8$ and 16 .

In Fig. 4 we have evaluated and compared the PAPR performance of the designed scheme for different number of sub-blocks $V=4,8$ and 16 . It is noticed that PAPR value at $\mathrm{CCDF}=10^{-3}$ equals to $8.3 \mathrm{~dB}$ for $V=4,7.2 \mathrm{~dB}$ for $V=8$ and 6.3 $\mathrm{dB}$ for $V=16$ whereas the original OFDM signal achieves this value at $11 \mathrm{~dB}$. As the value of sub-blocks $V$ increases, $\mathrm{t}$ we can notice a significant improvement in the PAPR performance of the OFDM signal. For more number of subblocks, the average iterations increase that further leads to higher number of computations in the designed scheme.

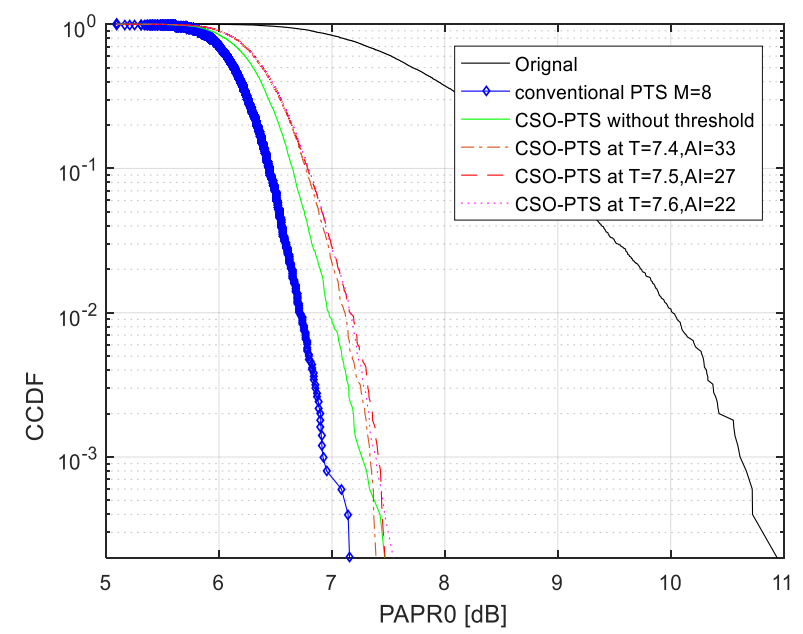

Fig. 5. PAPR performance of proposed scheme at threshold value $=7.4,7.5$ and 7.6.
Fig. 5 shows the PAPR performance of the designed CSOPTS systems for different threshold values $T=7.4,7.5$ and 7.6 $\mathrm{dB}$ using relevant algorithmic parameters. It is noticed that with a threshold value we can limit the number of average iterations (AI) as well as PAPR reduction capability in the designed system. Conventional PTS scheme needs 128 average iterations per OFDM frame to achieve $\mathrm{CCDF}=2 * 10^{-4}$ at about $7.3 \mathrm{~dB}$ but designed system can achieve same CCDF at $T=7.4 \mathrm{~dB}$ threshold using $A I=33$ average iterations per OFDM frame, at $T=7.5 \mathrm{~dB}$ threshold using $A I=27$ average iterations per OFDM frame, and at $T=7.6 \mathrm{~dB}$ threshold using only $A I=22$ average iterations per OFDM frame. Our designed system with threshold limit yields complexity reduction due to less number of average iterations as compared to the conventional PTS-OFDM systems. It can be seen from the plots that at lower values of threshold there is an improvement in the PAPR performance of the system but at the cost of computational complexity. High threshold values results in lesser number of search iterations. Thus, suitable selection of threshold value enables to achieve effective trade-offs between the PAPR reduction and computational complexity.

The CCDF of the proposed algorithms are simulated and compared with the conventional PTS-OFDM system in Fig. 6. It is studied from the results that the PAPR value of the original OFDM signal, proposed CSO-PTS without threshold and with threshold limits are approximately $11 \mathrm{~dB}, 7.23 \mathrm{~dB}$ and $7.35 \mathrm{~dB}$ for $\mathrm{CCDF}=10^{-3}$. These two proposed algorithm differ in the search computations. After the $\mathrm{CCDF}=10^{-3}$ the PAPR performance of the two proposed schemes nearly coincide with one another. It is also observed that in the proposed scheme less iterations are required at the cost of marginal performance loss of $0.28 \mathrm{~dB}$ in contrast to the conventional PTS-OFDM system having PAPR value as 7.07 $\mathrm{dB}$ at a given $\mathrm{CCDF}=10^{-3}$.

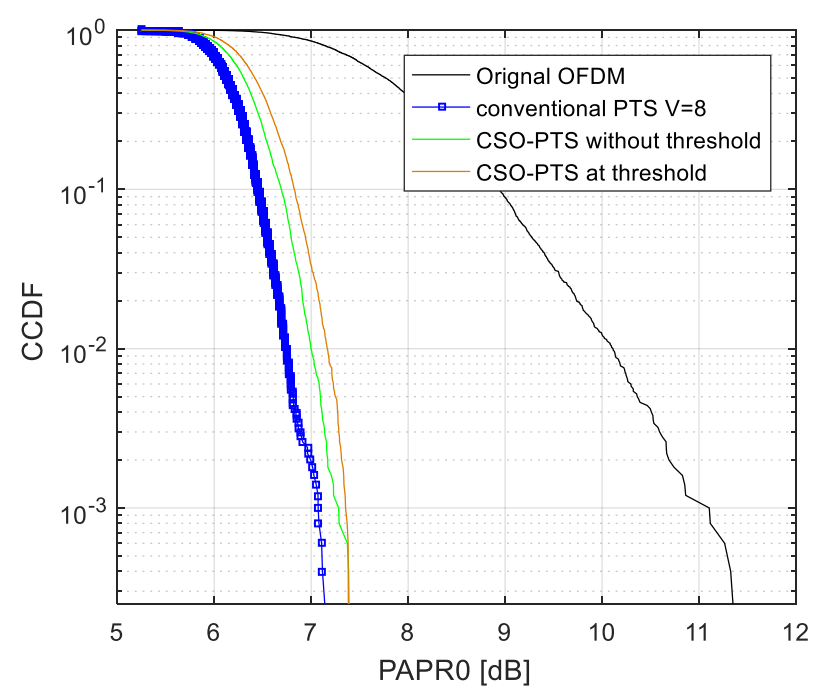

Fig. 6. PAPR comparison of the proposed algorithms without threshold and with threshold limit and the Conventional PTS-OFDM system. 
TABLE II

COMPARISONS OF THE AVERAGE ITERATIONS OF THE PROPOSED ALGORITHMS WITH CONVENTIONAL PTS-OFDM SYSTEM.

\begin{tabular}{|l|l|l|}
\hline Schemes & $\begin{array}{l}\text { Average } \\
\text { iterations (AI) }\end{array}$ & $\begin{array}{l}\text { PAPR(dB), } \\
\text { at CCDF }\left(10^{-3}\right)\end{array}$ \\
\hline Original OFDM & 0 & $11 \mathrm{~dB}$ \\
\hline Conventional PTS & 128 & $7.07 \mathrm{~dB}$ \\
\hline $\begin{array}{l}\text { CSO-PTS without } \\
\text { threshold }\end{array}$ & 67 & $7.23 \mathrm{~dB}$ \\
\hline CSO-PTS with threshold & 33 & $7.35 \mathrm{~dB}$ \\
\hline
\end{tabular}

Table II illustrates the comparative analysis of average iterations and PAPR performance of the proposed technique with the conventional PTS-OFDM systems. Observations are noted on the basis of relevant algorithmic parameters. It can be observed that the conventional PTS scheme needs 128 average iterations per OFDM frame for $V=8$. It is found that the CSO-PTS systems without threshold value requires 67 average iterations per OFDM frame and CSO-PTS systems with threshold needs 33 average iterations per OFDM frame for optimal search. In comparison to conventional PTS scheme the number of iterations required for optimal search are remarkably reduced at the cost of slight PAPR performance loss of approximately $0.28 \mathrm{~dB}$ at $\mathrm{CCDF}=10^{-3}$ respectively.

Table III depicts the comparative analysis of real additions, real multiplications and CCRR values of the proposed technique with the conventional PTS-OFDM systems. The number of computations are calculated using the expressions in (16), (17) and (18).

TABLE III

COMPARISONS OF THE COMPUTATIONAL COMPLEXITY OF THE PROPOSED ALGORITHMS WITH CONVENTONAL PTS-OFDM SYSTEM.

\begin{tabular}{|l|l|l|l|}
\hline Schemes & $\begin{array}{l}\text { Conventional } \\
\text { PTS }\end{array}$ & $\begin{array}{l}\text { CSO-PTS } \\
\text { without } \\
\text { threshold }\end{array}$ & $\begin{array}{l}\text { CSO-PTS } \\
\text { with threshold }\end{array}$ \\
\hline Real Additions & 540672 & 306432 & 16896 \\
\hline $\begin{array}{l}\text { Real } \\
\text { Multiplications }\end{array}$ & 98304 & 67072 & 49664 \\
\hline $\begin{array}{l}\text { CCRR } \\
(\%)\end{array}$ & $41.5 \%$ & $69.8 \%$ \\
\hline
\end{tabular}

It is noted from the table that for the same PAPR reduction, the value of CCRR in CSO-PTS systems without threshold is $41.5 \%$ while in CSO-PTS systems with threshold is $69 \%$ in respect to the conventional PTS scheme. Hence low computational complexity is obtained for both the Algorithms in our designed scheme when compared to the conventional PTS-OFDM system.

Fig 7. shows the BER performance of our proposed schemes in the AWGN and Rayleigh fading channel. In our simulations we have used a 3-tap Stanford University Interim (SUI) 5-multipath fading channel with average path gains [0 $\mathrm{dB},-5 \mathrm{~dB},-10 \mathrm{~dB}]$, and path delays $[0 \mu \mathrm{s}, 4 \mu \mathrm{s}, 10 \mu \mathrm{s}]$. It is noted from the curves that the BER degradations over multipath fading channel are more than that in the AWGN channel. It is observed from the curves that the OFDM system and the conventional PTS system have almost same BER degradations under perfect side information conditions. Also in our proposed schemes, we are using perfect side information at the receiver side, hence there is no performance loss in comparison to the conventional PTS scheme. Thus, the proposed scheme offers negligible loss in the BER performance of the OFDM system in the AWGN and Rayleigh fading channel.

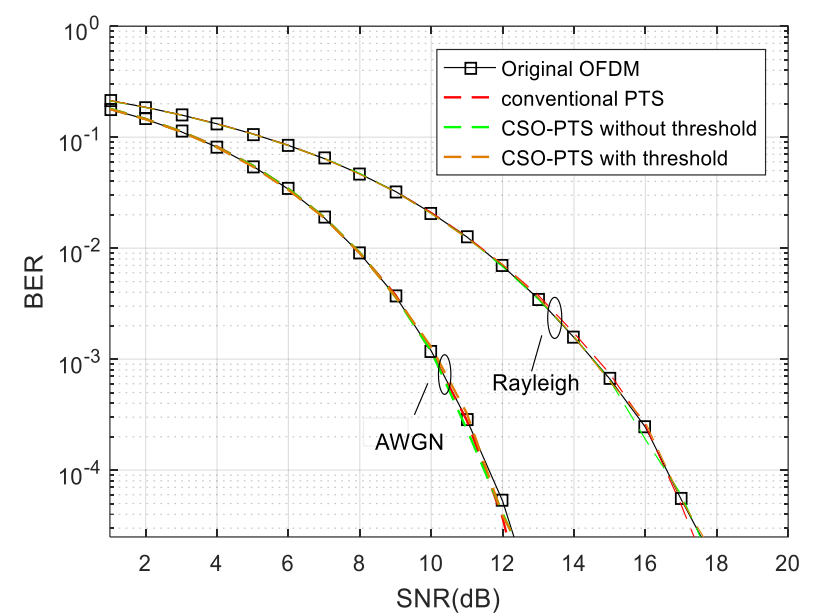

Fig. 7. BER vs SNR of the proposed algorithms over AWGN and Rayleigh fading channels.

\section{CONCLUSION}

In this paper, we have proposed two schemes for CSO based PTS system (i) CSO-PTS system without threshold (ii) CSO-PTS system with threshold limit. CSO algorithm has better convergence performance in terms of both speed and precision which are vital for finding an optimal solution. The designed schemes offer low computational complexity in terms of less average iterations per OFDM frame and high value of CCRR in comparison to the conventional PTS scheme. Improved PAPR reduction capability of the OFDM signal is achieved without any BER degradations over AWGN and fading channels. System performance is studied for different values of maximum generations, population size and threshold limits. It is observed that effective trade-offs between the PAPR reduction and computational complexity are obtained in our proposed schemes. An appropriate value of threshold limit in the designed CSO-PTS system remarkably reduces the number of average iterations with a slight loss in PAPR performance in comparison to conventional PTS system. Hence, our proposed schemes offer a significant reduction in complexity with PAPR reduction capability of the OFDM signal and negligible deterioration in the BER performance of the system. Hence, our proposed schemes offer a significant reduction in complexity with improved PAPR reduction capability of the OFDM signal and negligible deterioration in the BER performance of the system.

\section{REFERENCES}

[1] W. Y. Zou and Y. Wu, "COFDM: An overview," IEEE Trans. Broadcast.,vol. 41, no. 1, pp. 1-8, Mar. 1995 doi:10.1109/11.372015 
[2] J.Hou, J. Ge, D. Zhai, J. Li, "Peak-to-Average Power Ratio Reduction of OFDM Signals With Nonlinear Companding Scheme," IEEE Trans. Broadcast.,vol. 56, no. 2, pp. 258-262,June 2010. doi:10.1109/TBC.2010.2046970

[3] X. Li and L. J. Cimini, Jr, "Effects of clipping and filtering on the performance of OFDM," IEEE Communications Letters, vol. 2, no. 5, pp.131-133, May 1998. doi: 10.1109/4234.673657

[4] T. Jiang and G. X. Zhu, "Nonlinear companding transform for reducing peak-to-average power ratio of OFDM signals," IEEE Trans. Broadcasting, vol. 50, no. 3, pp. 342-346, Sep. 2004. doi:10.1109/TBC.2004.834030

[5] T. Jiang and G. Zhu, "Complement block coding for reduction in peakto-average power ratio of OFDM signals," IEEE Communications Magazine, vol. 43, no. 9, pp. S17-S22, Sep. 2005. doi 10.1109/MCOM.2005.1509967

[6] R. W. Bauml, R. F. H. Fisher, and J. B. Huber, "Reducing the Peak-toAverage Power Ratio of Multicarrier Modulation by Selected Mapping,"IEEE Electron. Lett., vol. 32, no. 22, pp. 2056-2057, Oct.1996. doi: 10.1049/el:19961384

[7] S. H. Muller and J. B. Huber, "OFDM with reduced peak-to-average power ratio by optimum combination of partial transmit sequences,' IEEE Electron. Lett, vol. 33, no. 5, pp. 36-69, Feb. 1997. doi:10.1049/el:19970266

[8] B.S. Krongold and D.L. Jones, "Peak Power Reduction via Active Constellation Extension," IEEE Trans. Broadcast.,vol. 49, no. 3, pp. 258-268, Sep. 2003. doi: 10.1109/TBC.2003.817088

[9] J. Tellado,"Multicarrier Transmission with low PAR." Ph.D.thesis, Stanford University, CA, 1998.

[10] S.H.Han, J.M.Cioffi, J.H.Lee, "Tone injection with hexagonal constellation for peak-to-average power ratio reduction in OFDM," IEEE Commun. Lett., vol. 10, no. 9, pp. 646-648, Sept. 2006. doi: 10.1109/LCOMM.2006.1714532

[11] C. Tellambura, "Improved phase factor computation for the PAR reduction of an OFDM signal using PTS," IEEE Commun. Lett., vol. 5 , no. 4, pp. 135-137, Apr. 2001. doi: 10.1109/4234.917092

[12] S. Das, P.N. Suganthan, Differential evolution: A survey of the stateof-the-art. IEEE Transactions on Evolutionary Computation vol. 15, no. 1, pp. 4-31, Feb 2011. doi: 10.1109/TEVC.2010.2059031

[13] J. K. Lain, S. Y. Wu, and P. H. Yang, "PAPR reduction of OFDM signal using PTS: A real-valued genetic approach," EURASIP J. Wireless Commun. Netw., vol. 2011, no. 126, pp. 1-8, Oct. 2011 doi:10.1186/1687-1499-2011-126

[14] F. S. Abu-Mouti and M. E. El-Hawary, "Optimal distributed generation allocation and sizing in distribution systems via artificial bee colony algorithm," IEEE Trans. Power Del., vol. 26, no. 4, pp. 2090-2101, Oct. 2011. doi: 10.1109/TPWRD.2011.2158246

[15] J. Zhou et al., "A Modified Shuffled Frog Leaping Algorithm for PAPR Reduction in OFDM Systems," IEEE Trans. Broadcast, vol. 61, no. 4, pp. 698-709, Dec. 2015.

[16] P. R. Kumar, P.V. Naganjaneyulu and K. S Prasad, "Hybrid PS-GW optimised PTS scheme for PAPR reduction in OFDM system" IET Communications, vol. 13, no.18, pp 2996-3002, Nov. 2019. doi:10.1049/iet-com.2019.0261

[17] J.H.Wen, S.-H.Lee, Y.-F.Huang, and H.-L.Hung, "A sub-optimal PTS algorithm based on particle swarm optimization technique for PAPR reduction in OFDM systems,"EURASIP J. Wirel. Commun. Netw., no. 1, pp.1-8, May 2008. doi: 10.1155/2008/601346

[18] T. Zhang, S. Li, and X. Yu, "Global Optimal Firefly Algorithm in Peakto-Average Power Ratio Reduction of Orthogonal Frequency Division Multiplexing Systems," Sensor Lett., vol. 12, no. 2, pp. 281-286, 2014 doi: 10.1166/sl.2014.3279

[19] R. Chauhan, N.Sood and I.Saini, "Galactic Swarm Optimization-PTS strategy to minimize PAPR in WP-OFDM system"in Proc. Int. Conf. on Intelligent Computing and Control Systems, 2019, pp 805-808. doi:10.1109/ICCS45141.2019.9065395

[20] X. Meng, Y. Liu, X. Gao, and H. Zhang, "A new bio- inspired algorithm: chicken swarm optimization," in Advances in Swarm Intelligence (Lecture Notes in Computer Science), vol. 8794. Cham, Switzerland: Springer,2014, pp. 86-94. doi:10.1007/978-3-319-118574_10

[21] D. Wu, S. Xu, and F. Kong, "Convergence analysis and improvement of the chicken swarm optimization algorithm," IEEE Access, vol. 4, pp. 9400- 9412, Sep 2016. doi: 10.1109/ACCESS.2016.2604738
[22] J. Wang, Z. Cheng, O. K. Ersoy, M. Zhang, K.Sun and Y. Bi "Improvement and Application of Chicken Swarm Optimization for Constrained Optimization," IEEE Access vol.7, pp. 58053-58072, March 2019. doi: 10.1109/ACCESS.2019.2913180

[23] Z.Yi, J. Liu, S. Wang, X. Zeng and J. Lu "PAPR Reduction Technology Based on CSO Algorithm in CO-OFDM System" in Proc. Int. Conf. on Optical Communications and Networks, China, 2016. doi:10.1109/ICOCN.2016.7875701

[24] A H M R. Awal, Z. Dou, Md. Al. Shayokh, Md. I. Zahoor "Implementation of chicken swarm optimization (CSO) with partial transmit sequences for the reduction of PAPR in OFDM system," in Proc. IEEE Int. Conf. on Communication Software and Networks, China, 2017, pp 468-472. doi:10.1109/ICCSN.2017.8230156

[25] H. Li, T. Jiang, and Y. Zhou, "A novel subblock linear combination scheme for peak-to-average power ratio reduction in OFDM systems," IEEE Trans. Broadcast.vol. 58 no. 3, pp. 360-369, Sep 2012. doi:10.1109/TBC.2012.2191698.

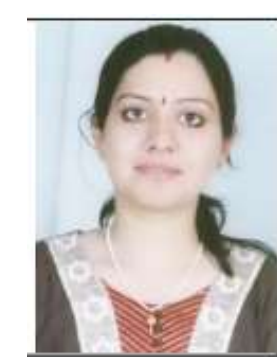

Saruti Gupta was born in Chandigarh,India.She received her B.Tech degree in Electronics and Communications Engineering from Swami Devi Dyal Institute of Engineering and Technology Panchkula, India in the year 2008. She received her M.Tech. in Electronics and Communications from Institute of Engineering and Technology, Bhaddal in the year 2013.Currently, she is persuing $\mathrm{PhD}$ degrees from Jaypee Institute of Information Technology, Noida. Her research interests include signal processing and PHY layer design for wireless communications.

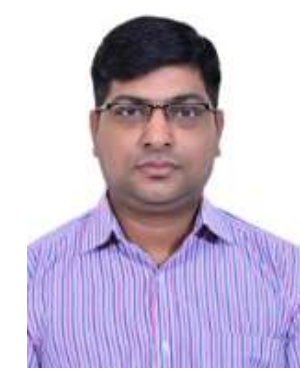

Ashish Goel was born in Rampur (U.P.), India. He received his B.Tech. degree in Electronics and Communications Engineering from Institute of Engineering Technology Bareilly, India in the year 2002. He received his M.Tech. in Information \& Communication Technology and $\mathrm{PhD}$ degrees from Jaypee Institute of Information Technology, Noida in the year 2004 and 2013 respectively. He joined, ECE department of Jaypee Institute of Information Technology, Noida as a faculty member in year 2004 and working as Associate Professor. He has published more than 25 research papers in reputed International Journals and Conferences. His research interests include high speed wireless communication systems, channel coding, MIMO-OFDM, Mobility Management and Cryptography. 